\title{
Endothelial Cell Metabolism in Atherosclerosis
}

\author{
Kosta Theodorou ${ }^{1}$ and Reinier A. Boon ${ }^{1,2,3 *}$ \\ ${ }^{1}$ Centre of Molecular Medicine, Institute of Cardiovascular Regeneration, Goethe-University, Frankfurt am Main, Germany, \\ ${ }^{2}$ German Center for Cardiovascular Research DZHK, Partner Site Rhine-Main, Berlin, Germany, ${ }^{3}$ Department of Physiology, \\ Amsterdam Cardiovascular Sciences, VU University Medical Center, Amsterdam, Netherlands
}

Atherosclerosis and its sequelae, such as myocardial infarction and stroke, are the leading cause of death worldwide. Vascular endothelial cells (EC) play a critical role in vascular homeostasis and disease. Atherosclerosis as well as its independent risk factors including diabetes, obesity, and aging, are hallmarked by endothelial activation and dysfunction. Metabolic pathways have emerged as key regulators of many EC functions, including angiogenesis, inflammation, and barrier function, processes which are deregulated during atherogenesis. In this review, we highlight the role of glucose, fatty acid, and amino acid metabolism in EC functions during physiological and pathological states, specifically atherosclerosis, diabetes, obesity and aging.

Keywords: endothelial cells, metabolism, atherosclerosis, inflammation, hyperglycemia, hyperlipidemia, aging

\section{OPEN ACCESS}

Edited by:

Salvatore Papa,

University of Leeds, United Kingdom

Reviewed by:

Lukas Tomas,

Lund University, Sweden

Miguel Sánchez-Álvarez,

Centro Nacional de Investigaciones

Cardiovasculares (CNIC), Spain

*Correspondence:

Reinier A. Boon

boon@med.uni-frankfurt.de

Specialty section:

This article was submitted to

Signaling,

a section of the journal

Frontiers in Cell and Developmental

Biology

Received: 25 May 2018

Accepted: 13 July 2018

Published: 07 August 2018

Citation:

Theodorou K and Boon RA (2018)

Endothelial Cell Metabolism in

Atherosclerosis.

Front. Cell Dev. Biol. 6:82.

doi: 10.3389/fcell.2018.00082

\section{INTRODUCTION}

Cardiovascular diseases (CVDs) are the leading cause of morbidity and mortality worldwide (World Health Organization, 2014). The underlying pathology of the majority of cardiovascular-related deaths is atherosclerosis, which is a lipid-driven chronic inflammatory disease of the medium- and large-sized arteries (Lusis, 2000). One of the earliest detectable changes in the development of atherosclerosis is endothelial cell (EC) activation and dysfunction at lesion-prone areas of the arterial vasculature (Hajra et al., 2000). These areas, particularly at bends and branch points, are characterized by ECs that display a pro-inflammatory, pro-thrombotic phenotype with a reduced barrier function, which is prompted by disturbed blood flow dynamics (Tabas et al., 2015). Moreover, subendothelial retention and modification of apolipoprotein B-containing lipoproteins, such as low-density lipoproteins (LDL), further activate ECs. This triggers an inflammatory response, leading to the recruitment of monocytes into the intima where they differentiate into macrophages and engulf modified lipoproteins to become foam cells. Progressed atherosclerotic lesions are characterized by a fibrous cap overlaying a lipid rich, necrotic core, and an accumulation of leukocytes in the lateral edges, which promote plaque instability by modulating EC phenotype and through proteolytic degradation of extracellular matrix (ECM) components. Unstable lesions may, upon rupture, lead to an atherothrombotic event, causing clinical events such as myocardial infarction or stroke. Alternatively, superficial intimal erosions, as a consequence of EC apoptosis and detachment, may also trigger an atherothrombotic event and its clinical sequelae (Quillard et al., 2015; Tabas et al., 2015).

Although initially believed to be an inert, semi-permeable barrier between blood constituents, and the underlying subendothelial tissues, the endothelium is now viewed as a metabolically active organ that plays a crucial role in vascular homeostasis and throughout the life history of atherosclerosis. In this review, we will focus on EC metabolism in health and atherosclerosis development. First, we will briefly review the three major metabolic pathways [i.e., glucose, fatty acids (FA) and amino acids (AA)] in ECs, followed by the role of EC metabolism in atherosclerosis. 


\section{ENDOTHELIAL CELL METABOLISM IN HEALTH}

To date, studies investigating EC metabolism have been limited to using cultured ECs, whose metabolism may be rewired because of in vitro conditions that do not fully recapitulate the in vivo environment (Hensley et al., 2016; Cantor et al., 2017). Nevertheless, great strides have been made in dissecting the roles of metabolism in EC functions by conditional targeting of key metabolic genes in vivo.

\section{Glucose Metabolism}

Glycolysis is the main energy supplier in cultured ECs, accounting for $\sim 75-85 \%$ of the total ATP production (Krützfeldt et al., 1990; De Bock et al., 2013). It has been estimated that isolated coronary microvascular ECs metabolize $\sim 99 \%$ of glucose into lactate under aerobic conditions, while only $0.04 \%$ is oxidized in the tricarboxylic acid (TCA) cycle (Krützfeldt et al., 1990). Indeed, inhibiting glycolysis using 2-deoxy-D-glucose induces EC cytotoxicity, indicating that glucose is essential for proper EC functioning and maintenance (Merchan et al., 2010).

Upon stimulation with pro-angiogenic signals, like vascular endothelial growth factor (VEGF) and fibroblast growth factor 2, ECs increase their glycolytic flux to support proliferation and migration (De Bock et al., 2013; Yu et al., 2017). In contrast, limiting glycolysis by pharmacological inhibition or genetic silencing of phosphofructokinase-2/fructose-2,6-biphosphatase 3 (PFKFB3) or silencing of hexokinase (HK)2 impairs EC proliferation, migration and vascular sprouting (De Bock et al., 2013; Schoors et al., 2014; Yu et al., 2017). Moreover, endothelial-specific PFKFB3 or HK2 deficiency causes vascular hypobranching in mice (De Bock et al., 2013; Yu et al., 2017).

Apart from using glucose for energy production, there are also alternative fates for glucose in ECs (Lunt and Vander Heiden, 2011; Figure 1). After glucose is taken up by ECs through facilitated diffusion by the glucose transporters (GLUT), primarily by GLUT1, glucose is phosphorylated by HK to glucose-6-phosphate (G6P) (Mann et al., 2003; Lunt and Vander Heiden, 2011). G6P can be converted to and stored as glycogen or processed in the oxidative branch of the pentose phosphate pathway (oxPPP) to yield nicotinamide adenine dinucleotide phosphate (NADPH) and ribose-5-phosphate (R5P), which are used for antioxidant defense and nucleotide biosynthesis, respectively (Lunt and Vander Heiden, 2011; Adeva-Andany et al., 2016). Silencing of the G6P dehydrogenase (G6PD), the rate-limiting enzymes of oxPPP, reduces EC proliferation and migration, and increases cellular reactive oxygen species (ROS) (Leopold et al., 2003). Furthermore, ECs contain glycogen reservoirs and pharmacological inhibition of glycogen phosphorylase, the ratelimiting enzyme in glycogen degradation, impairs EC migration and viability (Vizán et al., 2009). However, how glycogen metabolism affects EC functions remains to be determined.

G6P can also be further metabolized to fructose-6-phosphate (F6P), which can be shunted into the hexosamine biosynthesis pathway (HBP) to produce UDP-N-acetylglucosamine (UDPGlcNAc), an important substrate for protein O-linked glycosylation that is critical for a plethora of EC functions (Laczy et al., 2009). Silencing of the rate-limiting enzyme in the HBP, glutamine:fructose-6-phosphate amidotransferase 1, augments VEGF-induced vascular sprouting (Zibrova et al., 2017). Furthermore, the two glycolysis intermediates F6P and glyceraldehyde-3-phosphate (G3P) can be used in the non-oxidative branch of the pentose phosphate pathway (nonoxPPP) to generate R5P, but not NADPH, in contrast to oxPPP (Lunt and Vander Heiden, 2011). Pharmacological inhibition of transketolase, the rate-limiting enzyme in non-oxPPP, limits ECs viability, and migration (Vizán et al., 2009).

ECs have a relatively low mitochondrial content $(<2-12 \%$ of the total cellular volume) compared to other cell types, such as hepatocytes (28\%) and cardiomyocytes (22-37\%, depending on the species; Blouin et al., 1977; Oldendorf et al., 1977; Barth et al., 1992). This is also reflected by the lower mitochondrial respiration of ECs compared to more oxidative cell types, like hepatocytes and cardiomyocytes (De Bock et al., 2013). Nevertheless, ECs have a considerable spare mitochondrial respiratory capacity (Doddaballapur et al., 2015; Wilhelm et al., 2016), which may be called upon during stress conditions like glucose deprivation to metabolize alternative substrates, such as FAs and glutamine (Krützfeldt et al., 1990; Mertens et al., 1990), however this remains to be determined. Cultured ECs derive only $\sim 15 \%$ of the total amount of ATP via oxidative pathways, suggesting that rather by playing a major role in energy production, mitochondria in ECs are more likely to have a signaling function (by producing ROS and maintaining intracellular $\mathrm{Ca}^{2+}$ homeostasis) and support biomass synthesis (by generating metabolic intermediates; Quintero et al., 2006; De Bock et al., 2013; Tang et al., 2014; Schoors et al., 2015; Huang et al., 2017).

\section{Fatty Acid Metabolism}

FAs in cultured ECs act as a carbon source for the production of the AA aspartate (a nucleotide precursor) and deoxyribonucleotides which are required for DNA synthesis, rather than supplying energy (accounting for $<5 \%$ of the total amount of ATP produced) or maintaining redox homeostasis (Figure 1; Schoors et al., 2015). Endothelialspecific deficiency or silencing of carnitine palmitoyltransferase (CPT)1A, the rate-limiting enzyme in FA oxidation (FAO), causes vascular sprouting defects in vivo and in vitro owing to a reduction in proliferation, but not migration (Schoors et al., 2015). In addition, pharmacological inhibition of CPT1 or silencing CPT1A or CPT2 reduces FAO and enhances endothelial permeability (Patella et al., 2015; Xiong et al., 2018). Furthermore, during lymphatic EC (LEC) differentiation, LECs upregulate CPT1A to support their proliferation, but also to promote their differentiation through acetyl-coenzyme A (acetyl-CoA) production, which is used for histone acetylation of lymphatic genes (Wong et al., 2017). Moreover, FAO maintains the cellular pool of acetyl-CoA and retains the identity of vascular ECs by reducing transforming growth factor $\beta$-induced endothelial-to-mesenchymal transition (EndMT) (Xiong et al., 2018). In addition to its role in EC proliferation, differentiation and permeability, FA metabolism also modulates the lipid 


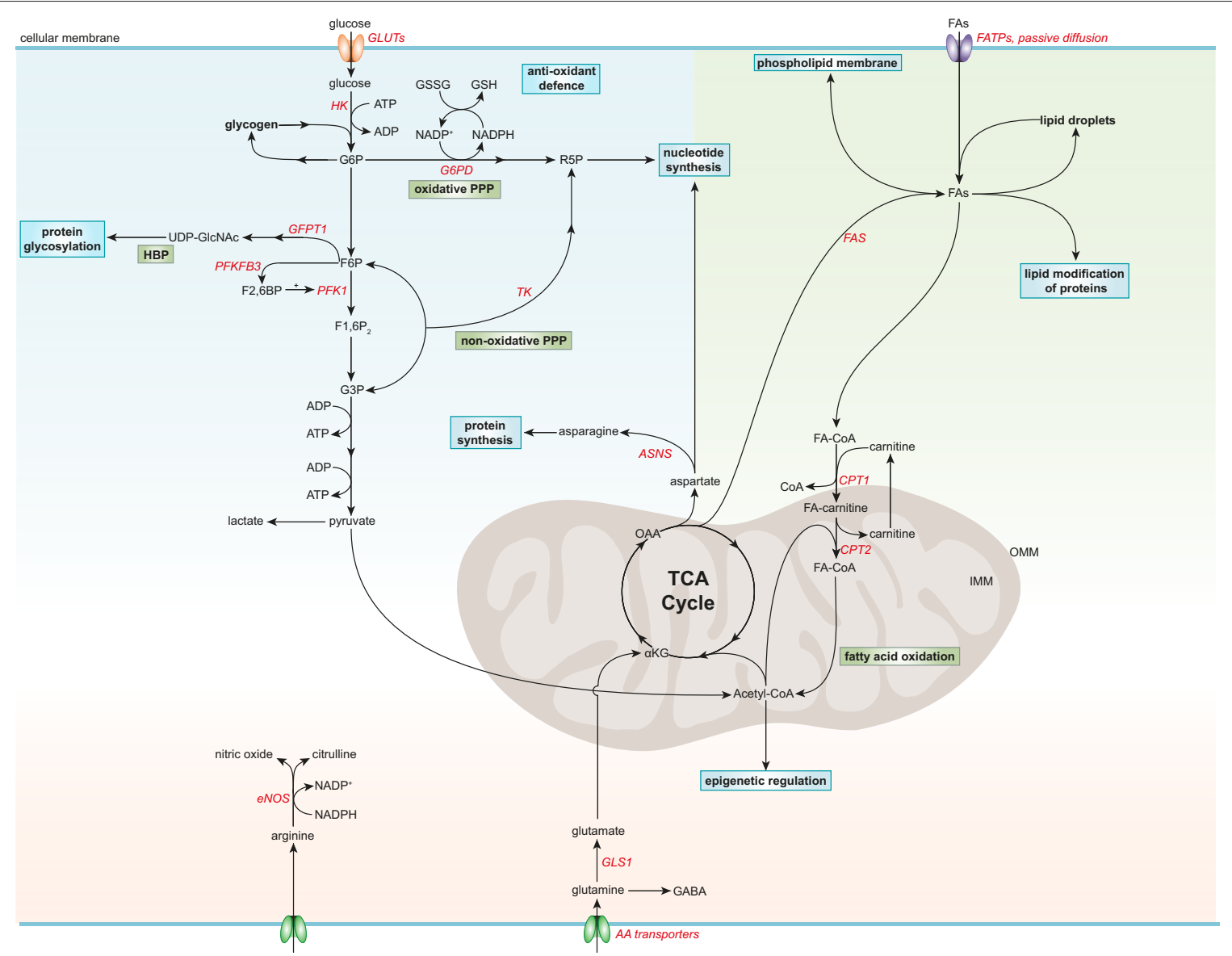

FIGURE 1 | Simplified general overview of glucose, fatty acid, and amino acid metabolism in healthy endothelial cells. Glucose enters ECs via glucose transporters (GLUTs), which is converted to glucose-6-phosphate (G6P) by hexokinase (HK) at the expense of adenosine triphosphate (ATP). G6P can be converted to glycogen for storage or used in the oxidative pentose phosphate pathway (PPP). The oxidative PPP generates reduced glutathione (GSH) and ribose-5-phosphate (R5P), which are used in the anti-oxidant defense and nucleotide synthesis, respectively. G6P can be further metabolized to fructose-6-phosphate (F6P) which is converted to uridine diphosphate $\mathrm{N}$-acetylglucosamine (UDP-GIcNAc), a substrate used for protein glycosylation, in the hexosamine biosynthesis pathway (HBP). F6P can also be converted to fructose-2,6-bisphophate (F2,6BP) by 6-phosphofructo-2-kinase/fructose-2,6-bisphosphatase 3 (PFKF3B), the main isoform in ECs. F2,6BP is a

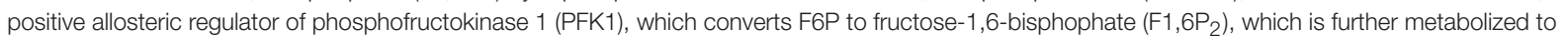
glyceraldehyde-3-phosphate (G3P). G3P together with F6P can be used in the non-oxidative PPP by transketolase (TK) to eventually produce R5P. G3P can also undergo several conversion steps leading to the production of ATP from adenosine diphosphate (ADP) and pyruvate, which can be further metabolized to lactate. After being taken up by fatty acid (FA) transporters (FATPs) or through passive diffusion, FAs become metabolically activated by coupling to coenzyme A (CoA). For FA oxidation (FAO) to occur, FAs have to be imported into the mitochondria, which is carried out by the acyl-carnitine shuttle system. At the outer mitochondrial membrane (OMM), carnitine palmitoyltransferase 1 (CPT1) converts FA-CoAs into FA-carnitines which facilitates the transport of FAs across the inner mitochondrial membrane (IMM) where they are converted back to FA-CoA by CPT2 (located in the IMM). FAO generates ATP and acetyl-CoA which is used in the tricarboxylic acid cycle (TCA). TCA intermediates are used for nucleotide synthesis or FA production, facilitated by fatty acid synthase (FAS). FAs can be stored in lipid droplets, converted to phospholipids to maintain the cell membrane or used for lipid modification of proteins. The amino acid (AA) glutamine is the most consumed AA in ECs. Once inside the cell, glutamine is converted to glutamate by glutaminase 1 (GLS1), which is further metabolized to $\alpha$-ketoglutarate ( $\alpha$ KG), a key intermediate in the TCA cycle. Through several metabolic steps, $\alpha$ KG is converted to oxaloacetic acid (OAA), which can be used for the generation of the AA aspartate. Aspartate can be used as a precursor for nucleotide synthesis or converted to the AA asparagine by asparagine synthetase (ASNS) using an ammonia group from glutamine thereby generating glutamate. Another important AA in ECs is arginine, which is used for the generation of the anti-atherogenic gaseous molecule nitric oxide using nicotinamide adenine dinucleotide phosphate (NADPH) as a cofactor. G6PD, glucose-6-phosphate dehydrogenase; GFPT1, glutamine:fructose-6-phosphate amidotransferase 1; GSSG, oxidized glutathione; $\mathrm{NADP}^{+}$, nicotinamide adenine dinucleotide.

composition of EC membranes, thereby regulating membrane stiffness and multiple cellular functions (Caires et al., 2017; Glatzel et al., 2018; Harayama and Riezman, 2018).

Besides using FAs for their own needs, ECs regulate the transport of FAs toward metabolically active tissues, such as skeletal and cardiac muscle (Mehrotra et al., 2014).
Circulating FAs, either bound to albumin or locally released from triglyceride-rich lipoproteins through lipoprotein lipasemediated lipolysis at the luminal surface of ECs, can enter ECs via passive diffusion or by FA transporter proteins. Interestingly, ECs readily store FAs in lipid droplets as a protective measure against endoplasmic reticulum (ER) stress (Kuo et al., 2017). 
Furthermore, FAs can be liberated from lipid droplets, which can be used by the ECs themselves or released to the underlying tissues (Kuo et al., 2017).

Although there is a continuous supply of FA from the blood stream that can enter cells, ECs also have the capability to synthesize FA de novo, since they express FA synthase (FAS; Wei et al., 2011; Hagberg et al., 2013). Silencing or genetic deletion of FAS impairs EC migration, vascular sprouting and permeability, and proper endothelial nitric oxide synthase (eNOS) functioning by reducing its palmitoylation (Wei et al., 2011).

\section{Amino Acid Metabolism}

Glutamine is the most consumed AA in ECs and is crucial for angiogenesis both in vitro and in vivo by contributing to TCA cycle anaplerosis, biomass synthesis and redox homeostasis (Huang et al., 2017; Kim B. et al., 2017). Withdrawal of glutamine, or pharmacological inhibition or knockdown of glutaminase 1 (GLS1), the rate-limiting enzyme in glutaminolysis, impairs EC proliferation, while the role of glutamine in EC migration remains controversial (Huang et al., 2017; Kim B. et al., 2017). Interestingly, supplementation of asparagine in glutamine-depleted conditions restores protein synthesis and EC function (Huang et al., 2017; Pavlova et al., 2018). Moreover, reducing glutamate-dependent asparagine synthesis by silencing asparagine synthase limits EC sprouting (Huang et al., 2017).

Arginine can be converted by eNOS to citrulline and nitric oxide (NO), an endogenous gaseous signaling molecule that has a wide variety of biological properties that maintain vascular homeostasis and are atheroprotective, such as suppression of thrombosis, inflammation and oxidative stress (Tousoulis et al., 2012).

Valine metabolism generates 3-hydroisobutyrate (3-HIB) which promotes transendothelial FA transport and skeletal muscle FA uptake and storage, however how valine and 3-HIB affect EC metabolism and function remains to be determined (Jang et al., 2016).

Furthermore, in vitro and in vivo restriction of sulfur AAs methionine and cysteine triggers an angiogenic response by promoting endothelial VEGF and hydrogen sulfide production thereby shifting EC metabolism from oxidative metabolism to glycolysis (Longchamp et al., 2018).

\section{ENDOTHELIAL CELL METABOLISM IN ATHEROSCLEROSIS}

\section{Endothelial Cell Activation by the Atherosclerotic Microenvironment}

ECs remain mostly quiescent throughout adult life, however, they can become activated in response to various physiological and pathological stimuli (Gimbrone and García-Cardeña, 2016; Figure 2). Disturbed blood flow dynamics are an important initiating factor of EC activation preceding atherogenesis (Hajra et al., 2000). High unidirectional laminar shear stress (LSS) activates an atheroprotective gene expression program in ECs, including the upregulation of transcription factor Krüppel-like factor 2 (KLF2; Dekker et al., 2002). KLF2 regulates a network of genes that maintain vascular barrier integrity and confer EC quiescence, resulting in an anti-inflammatory, anti-thrombotic EC phenotype (Dekker et al., 2006). Interestingly, high LSS suppresses EC glucose uptake, glycolysis and mitochondrial respiration via a KLF2-dependent mechanism (Doddaballapur et al., 2015).

In contrast, ECs at atheroprone areas of the vasculature are subject to disturbed low LSS and show activation of proinflammatory pathways and enhanced expression of glycolytic enzymes (Feng et al., 2017). Low LSS enhances EC glycolysis via a nuclear factor $\kappa \mathrm{B}(\mathrm{NF}-\kappa \mathrm{B})$-hypoxia inducible factor $1 \alpha-$ (HIF $1 \alpha)$-dependent axis, despite being in a highly oxygenated environment (Feng et al., 2017; Wu et al., 2017). Moreover, pro-inflammatory cytokines increase glucose uptake and glycolysis in ECs which augments cytokine-induced NF- $\mathrm{B}$ activation, most likely via lactate, however this requires further investigation (Folco et al., 2011; Végran et al., 2011; Cantelmo et al., 2016). Disturbed LSS and pro-inflammatory cytokines also activate the mechanotransducers Yes-associated protein (YAP) and transcriptional coactivator with PDZ-binding motif (TAZ), which promote a pro-inflammatory EC phenotype and atherosclerosis development (Wang K. C. et al., 2016; Wang L. et al., 2016b). Similar to NF-кB, YAP/TAZ signaling promotes EC glycolysis and vice versa (Enzo et al., 2015; Bertero et al., 2016; Kim J. et al., 2017). Partially blocking glycolysis by pharmacological inhibition of PFKFB3 reduces cancer cell adhesion to ECs and improves endothelial barrier function (Cantelmo et al., 2016), which may also limit leukocyte extravasation. Together, pro-inflammatory signaling enhances glycolysis and in turn glycolysis can drive pro-inflammatory programs, thereby forming a vicious cycle resulting in sustained pro-inflammatory signaling in ECs.

Furthermore, rupture-prone human atherosclerotic lesions are characterized by the presence of intraplaque neovascularization and hemorrhage (Virmani et al., 2005). Intraplaque Hypoxia and hemoglobin:haptoglobin complexes activate HIF $1 \alpha$-dependent signaling in macrophages leading to enhanced VEGF secretion, which in turn increases intraplaque angiogenesis, vascular permeability and leukocyte recruitment (Sluimer et al., 2008; Guo et al., 2018).

In contrast to glycolysis, the role of FAO in EC proinflammatory responses is not known. On the other hand, FAO maintains EC barrier function (Patella et al., 2015; Xiong et al., 2018). Furthermore, by undergoing EndMT, ECs contribute to the pool of mesenchymal cells within atherosclerotic lesions (Evrard et al., 2016). These EC-derived mesenchymal cells may contribute to plaque instability by enhanced expression and activity of matrix degrading proteins (Chen et al., 2015; Evrard et al., 2016). FAO has been shown to inhibit EndMT (Xiong et al., 2018), however, the role of glycolysis and AA metabolism herein remains to be determined. These studies suggest that endothelial FAO may reduce atherosclerosis development.

Besides its role in glycolysis, the pro-inflammatory YAP/TAZ pathway enhances EC glutaminolysis, suggesting a proatherogenic role for glutamine in ECs (Bertero et al., 2016). Interestingly, pharmacological inhibition of GLS1 does not 


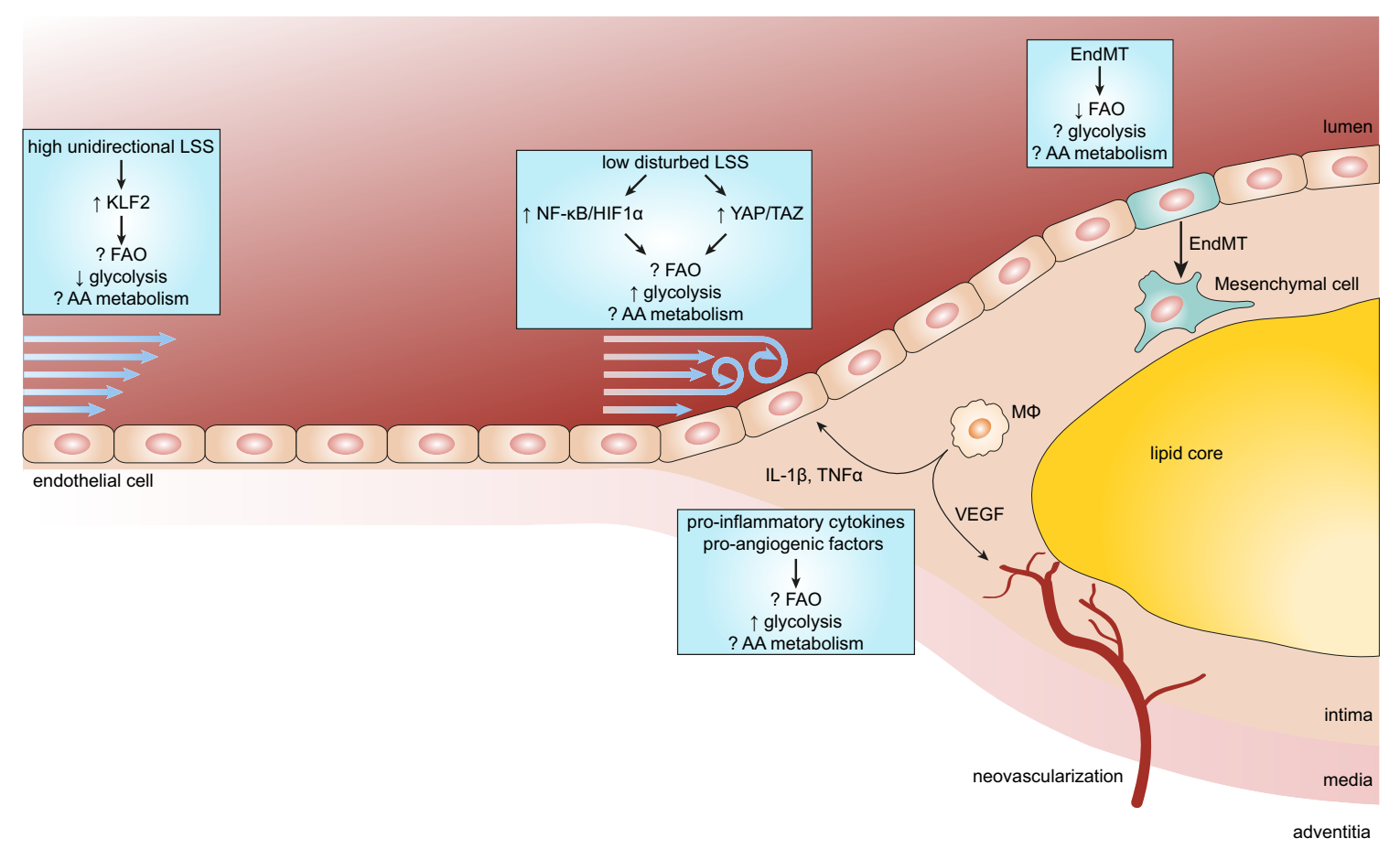

FIGURE 2 | Endothelial metabolism in atherosclerosis. Endothelial cells (ECs) exposed to high unidirectional laminar shear stress (LSS) activate atheroprotective signaling via the transcription factor Krüppel-like factor 2 (KLF2) which reduces glycolysis and maintains ECs in a quiescent state. In contrast, atheroprone regions are subjected to low disturbed LSS enhances EC glycolysis via the nuclear factor $\kappa B(N F-\kappa B) /$ hypoxia inducible factor $1 \alpha(H I F 1 \alpha)$ axis and mechanotransducers Yes-associated protein (YAP) and transcriptional coactivator with PDZ-binding motif (TAZ). During the process of atherosclerosis, ECs are exposed to a pro-inflammatory milieu [e.g., cytokines interleukin $1 \beta(\mathrm{IL}-1 \beta)$ and tumor necrosis factor $\alpha$ (TNF $\alpha)$ ], which also enhances glycolysis in ECs. In more progressed lesions, macrophages $(\mathrm{M} \Phi)$ start to secrete pro-angiogenic factors resulting in intraplaque neovascularization. Pro-angiogenic factors, like vascular endothelial growth factor (VEGF), enhance glycolysis in ECs to support proliferation and migration. In addition, reduced FAO in ECs predisposes them to undergo endothelial-to-mesenchymal transition (EndMT), which may affect plaque stability.

affect pro-inflammatory gene expression in or leukocyte adhesion on ECs. Glutamine starvation, however, reduces protein synthesis resulting in ER stress which stimulates proinflammatory signaling and apoptosis (Tabas, 2010; Huang et al., 2017). In addition, glutamine deficiency reduces nucleotide synthesis and increased ROS production, resulting in reduced proliferation and increased apoptosis, respectively (Huang et al., 2017). Moreover, ECs have been shown to synthesize the neurotransmitter $\gamma$-aminobutyric acid from glutamate, which exerts anti-inflammatory effects in ECs (Sen et al., 2016). Therefore, it remains to be determined how EC glutaminolysis affects atherosclerosis development.

Interestingly, statins, the cholesterol-lowering drugs which are the cornerstone of atherosclerosis treatment, have been shown to increase KLF2 expression, while reducing pro-inflammatory signaling by NF- $\kappa$ B, HIF1 $\alpha$, and YAP/TAZ (Dichtl et al., 2003; Sen-Banerjee et al., 2005; Wang L. et al., 2016b), suggesting that statins may also exert their anti-atherogenic effects by reprogramming EC metabolism.

\section{Deregulated Endothelial Cell Metabolism by Risk Factors for Atherosclerosis}

Risk factors, such as diabetes, obesity, and aging, have been shown to accelerate atherosclerosis development and are hallmarked by endothelial dysfunction and deregulated EC metabolism (Kanter et al., 2007; Wang and Bennett, 2012).

Diabetes is characterized by high glucose levels in the circulation which increases endothelial ROS production through auto-oxidation of glucose, NADPH-oxidases, eNOS uncoupling, and mitochondrial dysfunction, leading to DNA damage and subsequent activation of poly(ADP-ribose) polymerase 1 (PARP1) (Du et al., 2003; Forrester et al., 2018). ADP-ribosylation by PARP1 inhibits the glycolytic enzyme glyceraldehyde-3-phosphate dehydrogenase (GAPDH) resulting in the accumulation of glycolytic intermediates upstream of GAPDH and their redistribution to side branches of the glycolytic pathway, leading to (1) uncontrolled protein glycation via HBP, (2) production of advanced glycation end products (AGEs) through the polyol and methylglyoxal pathways, and (3) increased protein kinase $\mathrm{C}$ (PKC) activation through de novo synthesis of diacylglycerol from G3P (Du et al., 2003; Shah and Brownlee, 2016). Glycation of eNOS inhibits its activation, while PKC inhibits insulin-mediated activation of eNOS and increases the expression of the vasoconstrictor endothelin-1 (Du et al., 2001; Li et al., 2013). Furthermore, AGEs induce EC dysfunction through modification of proteins and ECM components, and activation of the receptor for AGEs resulting in activation of the pro-inflammatory NF- $\mathrm{B}$ signaling pathway, increased 
vascular leakage and ROS production (Shah and Brownlee, 2016). Interestingly, reverting the glucose intermediates F6P and G3P toward the PPP by activating transketolase using a thiamine derivative reduces all three hyperglycemia-induced pathways described above as well as NF- $\kappa$ B activity (Hammes et al., 2003).

Obesity, as well as diabetes, is associated with elevated circulating concentrations of saturated FAs and triglyceriderich lipoproteins, which can provide an additional supply of FAs (Goldberg and Bornfeldt, 2013; Nordestgaard, 2016). FAs can induce EC apoptosis and dysfunction by impairing NOmediated vasodilation and promoting vascular permeability, oxidative and ER stress, pro-inflammatory NF- $\mathrm{B}$ signaling and inflammasome activation (Inoguchi et al., 2000; Steinberg et al., 2000; Maloney et al., 2009; Tampakakis et al., 2016; Wang L. et al., 2016a). Reducing intracellular lipid levels by increasing FAO via peroxisome-proliferator-activated receptor (PPAR) $\beta$ and $\delta$-mediated upregulation of CPT1A or overexpression of PPAR- $\gamma$ coactivator $1-\alpha$ reduces FA-induced EC dysfunction and apoptosis, respectively (Won et al., 2010; Toral et al., 2015). Moreover, metformin, a first-line therapeutic drug against type 2 diabetes, decreases FA-induced ER stress and ROS production via a 5 ' adenosine monophosphate-activated protein kinase-PPAR $\delta$ dependent axis (Cheang et al., 2014). However, why ECs do not increase FAO naturally upon lipid overload, despite having a considerable spare mitochondrial respiratory capacity, remains to be explored.

Cellular aging is a complex process characterized by the progressive loss of cellular function and is hallmarked, among others, by deregulated nutrient sensing and mitochondrial dysfunction (López-Otín et al., 2013). Indeed, ECs from aged rats are characterized by a reduction in mitochondrial mass, altered expression of mitochondrial components and an increase in mitochondrial ROS production (Ungvari et al., 2007, 2008). Furthermore, nutrient sensing pathways are fundamental to the aging process, since dietary restriction protects against the aging-mediated decline in EC function (Csiszar et al., 2014). Interestingly, endothelial-specific overexpression of the $\mathrm{NAD}^{+}$dependent deacetylase sirtuin 1 , a sensor that detects energy availability via $\mathrm{NAD}^{+}$, or supplementation of NAD precursors

\section{REFERENCES}

Adeva-Andany, M. M., Gonzalez-Lucan, M., Donapetry-Garcia, C., FernandezFernandez, C., and Ameneiros-Rodriguez, E. (2016). Glycogen metabolism in humans. BBA Clin. 5, 85-100. doi: 10.1016/j.bbacli.2016.02.001

Barth, E., Stammler, G., Speiser, B., and Schaper, J. (1992). Ultrastructural quantitation of mitochondria and myofilaments in cardiac muscle from 10 different animal species including man. J. Mol. Cell. Cardiol. 24, 669-681. doi: 10.1016/0022-2828(92)93381-S

Bertero, T., Oldham, W. M., Cottrill, K. A., Pisano, S., Vanderpool, R. R., Yu, Q., et al. (2016). Vascular stiffness mechanoactivates YAP/TAZdependent glutaminolysis to drive pulmonary hypertension. J. Clin. Invest. 126, 3313-3335. doi: 10.1172/JCI86387

Blouin, A., Bolender, R. P., and Weibel, E. R. (1977). Distribution of organelles and membranes between hepatocytes and nonhepatocytes in the rat liver parenchyma. A stereological study. J. Cell Biol. 72, 441-455. doi: $10.1083 /$ jcb.72.2.441

Brunet, A., and Rando, T. A. (2017). Interaction between epigenetic and metabolism in aging stem cells. Curr. Opin. Cell Biol. 45, 1-7. doi: 10.1016/j.ceb.2016.12.009 reverse the aging-associated decline in angiogenesis (Das et al., 2018).

Furthermore, additional hallmarks of aging, such as epigenetic alterations, and cellular senescence, may also affect EC metabolism. For instance, aging-induced epigenetic modifications can regulate the expression of metabolic genes and, conversely, metabolic intermediates modulate the epigenetic landscape (Brunet and Rando, 2017). Replicative senescence in ECs, achieved through consecutive in vitro passaging, reduced ATP levels by $\sim 10$-fold, despite having enhanced glycolysis, the main energy supplier in ECs (Unterluggauer et al., 2008; De Bock et al., 2013). Furthermore, inhibition of glutaminolysis induces a senescent phenotype in ECs in vitro (Unterluggauer et al., 2008). However, it remains to be determined which metabolic pathways are modulated by aging in ECs and whether EC metabolism can be targeted to reverse the aging-associated EC dysfunction.

\section{CONCLUDING REMARKS}

Metabolic pathways have emerged as key regulators of many EC functions, including angiogenesis, inflammation, and barrier function. However, despite major advances in our understanding in EC metabolism, there still remain many unanswered questions. Although research in endothelial AA metabolism is still in its infancy, limiting glycolysis and/or stimulating FAO in ECs may be a promising therapeutic strategy against atherosclerosis, even in the presence of risk factors such as diabetes and obesity. However, the majority of the complications due to atherosclerosis occur in the aged population (Shih et al., 2011). Therefore, a better understanding of the metabolic perturbations in aged ECs may provide additional therapeutic avenues in the treatment of atherosclerosis.

\section{AUTHOR CONTRIBUTIONS}

All authors listed have made a substantial, direct and intellectual contribution to the work, and approved it for publication.

Caires, R., Sierra-Valdez, F. J., Millet, J. R. M., Herwig, J. D., Roan, E., Vasquez, V., et al. (2017). Omega-3 fatty acids modulate TRPV4 function through plasma membrane remodeling. Cell Rep. 21, 246-258. doi: 10.1016/j.celrep.2017.09.029

Cantelmo, A. R., Conradi, L. C., Brajic, A., Goveia, J., Kalucka, J., Pircher, A., et al. (2016). Inhibition of the glycolytic activator PFKFB3 in endothelium induces tumor vessel normalization, impairs metastasis, and improves chemotherapy. Cancer Cell 30, 968-985. doi: 10.1016/j.ccell.2016.10.006

Cantor, J. R., Abu-Remaileh, M., Kanarek, N., Freinkman, E., Gao, X., Louissaint, A. Jr., et al. (2017). Physiologic medium rewires cellular metabolism and reveals uric acid as an endogenous inhibitor of UMP synthase. Cell 169, 258.e17-272.e17. doi: 10.1016/j.cell.2017.03.023

Cheang, W. S., Tian, X. Y., Wong, W. T., Lau, C. W., Lee, S. S., Chen, Z. Y., et al. (2014). Metformin protects endothelial function in dietinduced obese mice by inhibition of endoplasmic reticulum stress through 5 ' adenosine monophosphate-activated protein kinase-peroxisome proliferatoractivated receptor delta pathway. Arterioscler. Thromb. Vasc. Biol. 34, 830-836. doi: 10.1161/ATVBAHA.113.301938

Chen, P. Y., Qin, L., Baeyens, N., Li, G., Afolabi, T., Budatha, M., et al. (2015). Endothelial-to-mesenchymal transition drives atherosclerosis progression. J. Clin. Invest. 125, 4514-4528. doi: 10.1172/JCI82719 
Csiszar, A., Gautam, T., Sosnowska, D., Tarantini, S., Banki, E., Tucsek, Z., et al. (2014). Caloric restriction confers persistent anti-oxidative, pro-angiogenic, and anti-inflammatory effects and promotes anti-aging miRNA expression profile in cerebromicrovascular endothelial cells of aged rats. Am. J. Physiol. Heart Circ. Physiol. 307, H292-H306. doi: 10.1152/ajpheart.00307.2014

Das, A., Huang, G. X., Bonkowski, M. S., Longchamp, A., Li, C., Schultz, M. B., et al. (2018). Impairment of an endothelial $\mathrm{NAD}(+)-\mathrm{H}_{2} \mathrm{~S}$ signaling network is a reversible cause of vascular aging. Cell 173, 74.e20-89.e20. doi: 10.1016/j.cell.2018.02.008

De Bock, K., Georgiadou, M., Schoors, S., Kuchnio, A., Wong, B. W., Cantelmo, A. R., et al. (2013). Role of PFKFB3-driven glycolysis in vessel sprouting. Cell 154, 651-663. doi: 10.1016/j.cell.2013.06.037

Dekker, R. J., Boon, R. A., Rondaij, M. G., Kragt, A., Volger, O. L., Elderkamp, Y. W., et al. (2006). KLF2 provokes a gene expression pattern that establishes functional quiescent differentiation of the endothelium. Blood 107, 4354-4363. doi: 10.1182/blood-2005-08-3465

Dekker, R. J., Van Soest, S., Fontijn, R. D., Salamanca, S., De Groot, P. G., Vanbavel, E., et al. (2002). Prolonged fluid shear stress induces a distinct set of endothelial cell genes, most specifically lung Kruppel-like factor (KLF2). Blood 100, 1689-1698. doi: 10.1182/blood-2002-01-0046

Dichtl, W., Dulak, J., Frick, M., Alber, H. F., Schwarzacher, S. P., Ares, M. P., et al. (2003). HMG-CoA reductase inhibitors regulate inflammatory transcription factors in human endothelial and vascular smooth muscle cells. Arterioscler. Thromb. Vasc. Biol. 23, 58-63. doi: 10.1161/01.ATV.0000043456.48735.20

Doddaballapur, A., Michalik, K. M., Manavski, Y., Lucas, T., Houtkooper, R. H., You, X., et al. (2015). Laminar shear stress inhibits endothelial cell metabolism via KLF2-mediated repression of PFKFB3. Arterioscler. Thromb. Vasc. Biol. 35, 137-145. doi: 10.1161/ATVBAHA.114.304277

Du, X. L., Edelstein, D., Dimmeler, S., Ju, Q., Sui, C., and Brownlee, M. (2001). Hyperglycemia inhibits endothelial nitric oxide synthase activity by posttranslational modification at the Akt site. J. Clin. Invest. 108, 1341-1348. doi: 10.1172/JCI11235

Du, X., Matsumura, T., Edelstein, D., Rossetti, L., Zsengeller, Z., Szabo, C., et al. (2003). Inhibition of GAPDH activity by poly(ADP-ribose) polymerase activates three major pathways of hyperglycemic damage in endothelial cells. J. Clin. Invest. 112, 1049-1057. doi: 10.1172/JCI18127

Enzo, E., Santinon, G., Pocaterra, A., Aragona, M., Bresolin, S., Forcato, M., et al. (2015). Aerobic glycolysis tunes YAP/TAZ transcriptional activity. EMBO J. 34, 1349-1370. doi: 10.15252/embj.201490379

Evrard, S. M., Lecce, L., Michelis, K. C., Nomura-Kitabayashi, A., Pandey, G., Purushothaman, K. R., et al. (2016). Endothelial to mesenchymal transition is common in atherosclerotic lesions and is associated with plaque instability. Nat. Commun. 7:11853. doi: 10.1038/ncomms11853

Feng, S., Bowden, N., Fragiadaki, M., Souilhol, C., Hsiao, S., Mahmoud, M., et al. (2017). Mechanical activation of hypoxia-inducible factor $1 \alpha$ drives endothelial dysfunction at atheroprone sites. Arterioscler. Thromb. Vasc. Biol. 37, 2087-2101. doi: 10.1161/ATVBAHA.117.309249

Folco, E. J., Sheikine, Y., Rocha, V. Z., Christen, T., Shvartz, E., Sukhova, G. K., et al. (2011). Hypoxia but not inflammation augments glucose uptake in human macrophages: implications for imaging atherosclerosis with 18 fluorine-labeled 2-deoxy-D-glucose positron emission tomography. J. Am. Coll. Cardiol. 58, 603-614. doi: 10.1016/j.jacc.2011.03.044

Forrester, S. J., Kikuchi, D. S., Hernandes, M. S., Xu, Q., and Griendling, K. K. (2018). Reactive oxygen species in metabolic and inflammatory signaling. Circ. Res. 122, 877-902. doi: 10.1161/CIRCRESAHA.117.311401

Gimbrone, M. A. Jr., and García-Cardeña, G. (2016). Endothelial cell dysfunction and the pathobiology of atherosclerosis. Circ. Res. 118, 620-636. doi: 10.1161/CIRCRESAHA.115.306301

Glatzel, D. K., Koeberle, A., Pein, H., Loser, K., Stark, A., Keksel, N., et al. (2018). Acetyl-CoA carboxylase 1 regulates endothelial cell migration by shifting the phospholipid composition. J. Lipid Res. 59, 298-311. doi: 10.1194/jlr.M080101

Goldberg, I. J., and Bornfeldt, K. E. (2013). Lipids and the endothelium: bidirectional interactions. Curr. Atheroscler. Rep. 15:365. doi: 10.1007/s11883-013-0365-1

Guo, L., Akahori, H., Harari, E., Smith, S. L., Polavarapu, R., Karmali, V., et al. (2018). CD163+ macrophages promote angiogenesis and vascular permeability accompanied by inflammation in atherosclerosis. J. Clin. Invest. 128, 1106-1124. doi: 10.1172/JCI93025
Hagberg, C., Mehlem, A., Falkevall, A., Muhl, L., and Eriksson, U. (2013). Endothelial fatty acid transport: role of vascular endothelial growth factor B. Physiology 28, 125-134. doi: 10.1152/physiol.00042.2012

Hajra, L., Evans, A. I., Chen, M., Hyduk, S. J., Collins, T., and Cybulsky, M. I. (2000). The NF- $\kappa B$ signal transduction pathway in aortic endothelial cells is primed for activation in regions predisposed to atherosclerotic lesion formation. Proc. Natl. Acad. Sci. U.S.A. 97, 9052-9057. doi: 10.1073/pnas.97.16.9052

Hammes, H. P., Du, X., Edelstein, D., Taguchi, T., Matsumura, T., Ju, Q., et al. (2003). Benfotiamine blocks three major pathways of hyperglycemic damage and prevents experimental diabetic retinopathy. Nat. Med. 9, 294-299. doi: $10.1038 / \mathrm{nm} 834$

Harayama, T., and Riezman, H. (2018). Understanding the diversity of membrane lipid composition. Nat. Rev. Mol. Cell Biol. 19, 281-296. doi: 10.1038/nrm.2017.138

Hensley, C. T., Faubert, B., Yuan, Q., Lev-Cohain, N., Jin, E., Kim, J., et al. (2016). Metabolic heterogeneity in human lung tumors. Cell 164, 681-694. doi: 10.1016/j.cell.2015.12.034

Huang, H., Vandekeere, S., Kalucka, J., Bierhansl, L., Zecchin, A., Bruning, U., et al. (2017). Role of glutamine and interlinked asparagine metabolism in vessel formation. EMBO J. 36, 2334-2352. doi: 10.15252/embj.201695518

Inoguchi, T., Li, P., Umeda, F., Yu, H. Y., Kakimoto, M., Imamura, M., et al. (2000). High glucose level and free fatty acid stimulate reactive oxygen species production through protein kinase $\mathrm{C}$-dependent activation of NAD(P)H oxidase in cultured vascular cells. Diabetes 49, 1939-1945. doi: 10.2337/diabetes.49.11.1939

Jang, C., Oh, S. F., Wada, S., Rowe, G. C., Liu, L., Chan, M. C., et al. (2016). A branched-chain amino acid metabolite drives vascular fatty acid transport and causes insulin resistance. Nat. Med. 22, 421-426. doi: 10.1038/nm.4057

Kanter, J. E., Johansson, F., Leboeuf, R. C., and Bornfeldt, K. E. (2007). Do glucose and lipids exert independent effects on atherosclerotic lesion initiation or progression to advanced plaques? Circ. Res. 100, 769-781. doi: 10.1161/01.RES.0000259589.34348.74

Kim, B., Li, J., Jang, C., and Arany, Z. (2017). Glutamine fuels proliferation but not migration of endothelial cells. EMBO J. 36, 2321-2333. doi: 10.15252/embj.201796436

Kim, J., Kim, Y. H., Kim, J., Park, D. Y., Bae, H., Lee, D. H., et al. (2017). YAP/TAZ regulates sprouting angiogenesis and vascular barrier maturation. J. Clin. Invest. 127, 3441-3461. doi: 10.1172/JCI93825

Krützfeldt, A., Spahr, R., Mertens, S., Siegmund, B., and Piper, H. M. (1990). Metabolism of exogenous substrates by coronary endothelial cells in culture. J. Mol. Cell. Cardiol. 22, 1393-1404. doi: 10.1016/0022-2828(90)90984-A

Kuo, A., Lee, M. Y., and Sessa, W. C. (2017). Lipid droplet biogenesis and function in the endothelium. Circ. Res. 120, 1289-1297. doi: 10.1161/CIRCRESAHA.116.310498

Laczy, B., Hill, B. G., Wang, K., Paterson, A. J., White, C. R., Xing, D., et al. (2009). Protein O-GlcNAcylation: a new signaling paradigm for the cardiovascular system. Am. J. Physiol. Heart Circ. Physiol. 296, H13-H28. doi: 10.1152/ajpheart.01056.2008

Leopold, J. A., Walker, J., Scribner, A. W., Voetsch, B., Zhang, Y. Y., Loscalzo, A. J., et al. (2003). Glucose-6-phosphate dehydrogenase modulates vascular endothelial growth factor-mediated angiogenesis. J. Biol. Chem. 278, 32100-32106. doi: 10.1074/jbc.M301293200

Li, Q., Park, K., Li, C., Rask-Madsen, C., Mima, A., Qi, W., et al. (2013). Induction of vascular insulin resistance and endothelin-1 expression and acceleration of atherosclerosis by the overexpression of protein kinase C- $\beta$ isoform in the endothelium. Circ. Res. 113, 418-427. doi: 10.1161/CIRCRESAHA.113. 301074

Longchamp, A., Mirabella, T., Arduini, A., Macarthur, M. R., Das, A., TrevinoVillarreal, J. H., et al. (2018). Amino acid restriction triggers angiogenesis via gcn2/atf4 regulation of vegf and h2s production. Cell 173, 117.e14-129.e14. doi: 10.1016/j.cell.2018.03.001

López-Otín, C., Blasco, M. A., Partridge, L., Serrano, M., and Kroemer, G. (2013). The hallmarks of aging. Cell 153, 1194-1217. doi: 10.1016/j.cell.2013.05.039

Lunt, S. Y., and Vander Heiden, M. G. (2011). Aerobic glycolysis: meeting the metabolic requirements of cell proliferation. Annu. Rev. Cell Dev. Biol. 27, 441-464. doi: 10.1146/annurev-cellbio-092910-154237

Lusis, A. J. (2000). Atherosclerosis. Nature 407, 233-241. doi: 10.1038/35025203 
Maloney, E., Sweet, I. R., Hockenbery, D. M., Pham, M., Rizzo, N. O., Tateya, S., et al. (2009). Activation of NF-kappaB by palmitate in endothelial cells: a key role for NADPH oxidase-derived superoxide in response to TLR4 activation. Arterioscler. Thromb. Vasc. Biol. 29, 1370-1375. doi: 10.1161/ATVBAHA.109.188813

Mann, G. E., Yudilevich, D. L., and Sobrevia, L. (2003). Regulation of amino acid and glucose transporters in endothelial and smooth muscle cells. Physiol. Rev. 83, 183-252. doi: 10.1152/physrev.00022.2002

Mehrotra, D., Wu, J., Papangeli, I., and Chun, H. J. (2014). Endothelium as a gatekeeper of fatty acid transport. Trends Endocrinol. Metab. 25, 99-106. doi: 10.1016/j.tem.2013.11.001

Merchan, J. R., Kovacs, K., Railsback, J. W., Kurtoglu, M., Jing, Y., Pina, Y., et al. (2010). Antiangiogenic activity of 2-deoxy-D-glucose. PLoS ONE 5:e13699. doi: 10.1371 /journal.pone.0013699

Mertens, S., Noll, T., Spahr, R., Krutzfeldt, A., and Piper, H. M. (1990). Energetic response of coronary endothelial cells to hypoxia. Am. J. Physiol. 258, H689H694. doi: 10.1152/ajpheart.1990.258.3.H689

Nordestgaard, B. G. (2016). Triglyceride-rich lipoproteins and atherosclerotic cardiovascular disease: new insights from epidemiology, genetics, and biology. Circ. Res. 118, 547-563. doi: 10.1161/CIRCRESAHA.115. 306249

Oldendorf, W. H., Cornford, M. E., and Brown, W. J. (1977). The large apparent work capability of the blood-brain barrier: a study of the mitochondrial content of capillary endothelial cells in brain and other tissues of the rat. Ann. Neurol. 1, 409-417. doi: 10.1002/ana.410010502

Patella, F., Schug, Z. T., Persi, E., Neilson, L. J., Erami, Z., Avanzato, D., et al. (2015). Proteomics-based metabolic modeling reveals that fatty acid oxidation (FAO) controls endothelial cell (EC) permeability. Mol. Cell. Proteomics 14, 621-634. doi: 10.1074/mcp.M114.045575

Pavlova, N. N., Hui, S., Ghergurovich, J. M., Fan, J., Intlekofer, A. M., White, R. M., et al. (2018). As extracellular glutamine levels decline, asparagine becomes an essential amino acid. Cell Metab. 27, 428.e5-438.e5. doi: 10.1016/j.cmet.2017.12.006

Quillard, T., Araujo, H. A., Franck, G., Shvartz, E., Sukhova, G., and Libby, P. (2015). TLR2 and neutrophils potentiate endothelial stress, apoptosis and detachment: implications for superficial erosion. Eur. Heart J. 36, 1394-1404. doi: 10.1093/eurheartj/ehv044

Quintero, M., Colombo, S. L., Godfrey, A., and Moncada, S. (2006). Mitochondria as signaling organelles in the vascular endothelium. Proc. Natl. Acad. Sci. U.S.A. 103, 5379-5384. doi: 10.1073/pnas.0601026103

Schoors, S., Bruning, U., Missiaen, R., Queiroz, K. C., Borgers, G., Elia, I., et al. (2015). Fatty acid carbon is essential for dNTP synthesis in endothelial cells. Nature 520, 192-197. doi: 10.1038/nature14362

Schoors, S., De Bock, K., Cantelmo, A. R., Georgiadou, M., Ghesquiere, B., Cauwenberghs, S., et al. (2014). Partial and transient reduction of glycolysis by PFKFB3 blockade reduces pathological angiogenesis. Cell Metab. 19, 37-48. doi: 10.1016/j.cmet.2013.11.008

Sen, S., Roy, S., Bandyopadhyay, G., Scott, B., Xiao, D., Ramadoss, S., et al. (2016). Gamma-aminobutyric acid is synthesized and released by the endothelium: potential implications. Circ. Res. 119, 621-634. doi: 10.1161/CIRCRESAHA.116.308645

Sen-Banerjee, S., Mir, S., Lin, Z., Hamik, A., Atkins, G. B., Das, H., et al. (2005). Kruppel-like factor 2 as a novel mediator of statin effects in endothelial cells. Circulation 112, 720-726. doi: 10.1161/CIRCULATIONAHA.104. 525774

Shah, M. S., and Brownlee, M. (2016). Molecular and cellular mechanisms of cardiovascular disorders in diabetes. Circ. Res. 118, 1808-1829. doi: 10.1161/CIRCRESAHA.116.306923

Shih, H., Lee, B., Lee, R. J., and Boyle, A. J. (2011). The aging heart and post-infarction left ventricular remodeling. J. Am. Coll. Cardiol. 57, 9-17. doi: 10.1016/j.jacc.2010.08.623

Sluimer, J. C., Gasc, J. M., Van Wanroij, J. L., Kisters, N., Groeneweg, M., Sollewijn Gelpke, M. D., et al. (2008). Hypoxia, hypoxia-inducible transcription factor, and macrophages in human atherosclerotic plaques are correlated with intraplaque angiogenesis. J. Am. Coll. Cardiol. 51, 1258-1265. doi: 10.1016/j.jacc.2007.12.025

Steinberg, H. O., Paradisi, G., Hook, G., Crowder, K., Cronin, J., and Baron, A. D. (2000). Free fatty acid elevation impairs insulin-mediated vasodilation and nitric oxide production. Diabetes 49, 1231-1238 doi: $10.2337 /$ diabetes.49.7.1231

Tabas, I. (2010). The role of endoplasmic reticulum stress in the progression of atherosclerosis. Circ. Res. 107, 839-850. doi: 10.1161/CIRCRESAHA.110.224766

Tabas, I., Garcia-Cardena, G., and Owens, G. K. (2015). Recent insights into the cellular biology of atherosclerosis. J. Cell Biol. 209, 13-22. doi: $10.1083 /$ jcb.201412052

Tampakakis, E., Tabit, C. E., Holbrook, M., Linder, E. A., Berk, B. D., Frame, A. A., et al. (2016). Intravenous lipid infusion induces endoplasmic reticulum stress in endothelial cells and blood mononuclear cells of healthy adults. J. Am. Heart Assoc. 5:e02574. doi: 10.1161/JAHA.115.002574

Tang, X., Luo, Y. X., Chen, H. Z., and Liu, D. P. (2014). Mitochondria, endothelial cell function, and vascular diseases. Front. Physiol. 5:175. doi: 10.3389/fphys.2014.00175

Toral, M., Romero, M., Jimenez, R., Mahmoud, A. M., Barroso, E., GomezGuzman, M., et al. (2015). Carnitine palmitoyltransferase-1 up-regulation by PPAR-beta/delta prevents lipid-induced endothelial dysfunction. Clin. Sci. 129, 823-837. doi: 10.1042/CS20150111

Tousoulis, D., Kampoli, A. M., Tentolouris, C., Papageorgiou, N., and Stefanadis, C. (2012). The role of nitric oxide on endothelial function. Curr. Vasc. Pharmacol. 10, 4-18. doi: 10.2174/157016112798829760

Ungvari, Z., Labinskyy, N., Gupte, S., Chander, P. N., Edwards, J. G., and Csiszar, A. (2008). Dysregulation of mitochondrial biogenesis in vascular endothelial and smooth muscle cells of aged rats. Am. J. Physiol. Heart Circ. Physiol. 294, H2121-H2128. doi: 10.1152/ajpheart.00012.2008

Ungvari, Z., Orosz, Z., Labinskyy, N., Rivera, A., Xiangmin, Z., Smith, K., et al. (2007). Increased mitochondrial $\mathrm{H}_{2} \mathrm{O}_{2}$ production promotes endothelial NF-кB activation in aged rat arteries. Am. J. Physiol. Heart Circ. Physiol. 293, H37-H47. doi: 10.1152/ajpheart.01346.2006

Unterluggauer, H., Mazurek, S., Lener, B., Hutter, E., Eigenbrodt, E., Zwerschke, W., et al. (2008). Premature senescence of human endothelial cells induced by inhibition of glutaminase. Biogerontology 9, 247-259. doi: $10.1007 /$ s10522-008-9134-x

Végran, F., Boidot, R., Michiels, C., Sonveaux, P., and Feron, O. (2011). Lactate influx through the endothelial cell monocarboxylate transporter MCT1 supports an NF-kappaB/IL-8 pathway that drives tumor angiogenesis. Cancer Res. 71, 2550-2560. doi: 10.1158/0008-5472.CAN-10-2828

Virmani, R., Kolodgie, F. D., Burke, A. P., Finn, A. V., Gold, H. K., Tulenko, T. N., et al. (2005). Atherosclerotic plaque progression and vulnerability to rupture: angiogenesis as a source of intraplaque hemorrhage. Arterioscler. Thromb. Vasc. Biol. 25, 2054-2061. doi: 10.1161/01.ATV.0000178991.71 605.18

Vizán, P., Sanchez-Tena, S., Alcarraz-Vizan, G., Soler, M., Messeguer, R., Pujol, M. D., et al. (2009). Characterization of the metabolic changes underlying growth factor angiogenic activation: identification of new potential therapeutic targets. Carcinogenesis 30, 946-952. doi: 10.1093/carcin/bgp083

Wang, J. C., and Bennett, M. (2012). Aging and atherosclerosis: mechanisms, functional consequences, and potential therapeutics for cellular senescence. Circ. Res. 111, 245-259. doi: 10.1161/CIRCRESAHA.111.261388

Wang, K. C., Yeh, Y. T., Nguyen, P., Limqueco, E., Lopez, J., Thorossian, S., et al. (2016). Flow-dependent YAP/TAZ activities regulate endothelial phenotypes and atherosclerosis. Proc. Natl. Acad. Sci. U.S.A. 113, 11525-11530. doi: $10.1073 /$ pnas. 1613121113

Wang, L., Chen, Y., Li, X., Zhang, Y., Gulbins, E., and Zhang, Y. (2016a). Enhancement of endothelial permeability by free fatty acid through lysosomal cathepsin B-mediated Nlrp3 inflammasome activation. Oncotarget 7, 73229-73241. doi: 10.18632/oncotarget.12302

Wang, L., Luo, J. Y., Li, B., Tian, X. Y., Chen, L. J., Huang, Y., et al. (2016b). Integrin-YAP/TAZ-JNK cascade mediates atheroprotective effect of unidirectional shear flow. Nature 540, 579-582. doi: 10.1038/nature20602

Wei, X., Schneider, J. G., Shenouda, S. M., Lee, A., Towler, D. A., Chakravarthy, M. V., et al. (2011). De novo lipogenesis maintains vascular homeostasis through endothelial nitric-oxide synthase (eNOS) palmitoylation. J. Biol. Chem. 286, 2933-2945. doi: 10.1074/jbc.M110.193037

Wilhelm, K., Happel, K., Eelen, G., Schoors, S., Oellerich, M. F., Lim, R., et al. (2016). FOXO1 couples metabolic activity and growth state in the vascular endothelium. Nature 529, 216-220. doi: 10.1038/nature 16498 
Won, J. C., Park, J. Y., Kim, Y. M., Koh, E. H., Seol, S., Jeon, B. H., et al. (2010). Peroxisome proliferator-activated receptor-gamma coactivator 1-alpha overexpression prevents endothelial apoptosis by increasing ATP/ADP translocase activity. Arterioscler. Thromb. Vasc. Biol. 30, 290-297. doi: 10.1161/ATVBAHA.109.198721

Wong, B. W., Wang, X., Zecchin, A., Thienpont, B., Cornelissen, I., Kalucka, J., et al. (2017). The role of fatty acid beta-oxidation in lymphangiogenesis. Nature 542, 49-54. doi: 10.1038/nature21028

World Health Organization (2014). Global Status Report on Noncommunicable Diseases 2014. Geneva: World Health Organization.

Wu, D., Huang, R. T., Hamanaka, R. B., Krause, M., Oh, M. J., Kuo, C. H., et al. (2017). HIF-1alpha is required for disturbed flow-induced metabolic reprogramming in human and porcine vascular endothelium. Elife 6:e25217. doi: $10.7554 /$ eLife.25217

Xiong, J., Kawagishi, H., Yan, Y., Liu, J., Wells, Q. S., Edmunds, L. R., et al. (2018). A metabolic basis for endothelial-to-mesenchymal transition. Mol. Cell 69, 689.e7-698.e7. doi: 10.1016/j.molcel.2018. 01.010
Yu, P., Wilhelm, K., Dubrac, A., Tung, J. K., Alves, T. C., Fang, J. S., et al. (2017). FGF-dependent metabolic control of vascular development. Nature 545, 224-228. doi: 10.1038/nature22322

Zibrova, D., Vandermoere, F., Goransson, O., Peggie, M., Marino, K. V., Knierim, A., et al. (2017). GFAT1 phosphorylation by AMPK promotes VEGF-induced angiogenesis. Biochem. J. 474, 983-1001. doi: 10.1042/BCJ20 160980

Conflict of Interest Statement: The authors declare that the research was conducted in the absence of any commercial or financial relationships that could be construed as a potential conflict of interest.

Copyright $\odot 2018$ Theodorou and Boon. This is an open-access article distributed under the terms of the Creative Commons Attribution License (CC BY). The use, distribution or reproduction in other forums is permitted, provided the original author(s) and the copyright owner(s) are credited and that the original publication in this journal is cited, in accordance with accepted academic practice. No use, distribution or reproduction is permitted which does not comply with these terms. 